\title{
Three-Dimensional Modelling of EUVE Observations of the Io Plasma Torus
}

\author{
N. THOMAS, ${ }^{1}$ D. E. INNES, ${ }^{1}$ AND R. LIEU ${ }^{2}$ \\ ${ }^{1}$ Max-Planck-Institut für Aeronomie, D-37191 Katlenburg-Lindau, Germany \\ ${ }^{2}$ Center for EUV Astrophysics, 2150 Kittredge St., \\ University of California, Berkeley, CA 94720-5030, USA
}

First results from a 3-D model of EUVE observations of the Io Plasma Torus are reported. The semi-empirical model calculations follow a method previously used to describe visible and near-UV emissions. The extension to EUV wavelengths is described. Several EUV emissions have been successfully modelled although some discrepancies remain at this stage. Most EUV emissions peak at a jovicentric distance of $\approx 5.8 \mathrm{R}$. The observed dawn-dusk asymmetry of the torus was well fitted with a shift parameter $(\epsilon)$ of 0.03 . The modelling also indicates that optical depth effects need to be considered for several EUV emission lines.

\section{Introduction}

The first observations of the Io plasma torus (IPT) at extreme ultraviolet wavelengths (EUV) were made by the ultraviolet spectrometer (UVS) instrument on Voyager 1 (Broadfoot et al., 1979). The data showed strong emission from a ring of ionized sulphur and oxygen species which completely surrounded Jupiter at a distance of $\approx 5.9 R_{J}$ (1 Jupiter radius $\left(R_{J}\right)=7.1410^{7} \mathrm{~m}$ ). The spectra were obtained at rather poor resolution $(\lambda / \delta \lambda \approx 25)$ and were severely blended. However, emissions at $685 \AA\left(\mathrm{S}^{2+}\right), 833 \AA\left(\mathrm{O}^{+}\right.$ and $\left.\mathrm{O}^{2+}\right), 910 \AA\left(\mathrm{S}^{+}\right), 1020 \AA\left(\mathrm{S}^{2+}\right)$, and $1070 \AA\left(\mathrm{S}^{3+}\right)$ could be identified.

Recent developments in EUV instrumentation have resulted in observations of the IPT at spectral resolutions roughly 10 times superior to that of the Voyager UVS instrument. The Extreme Ultraviolet Explorer (EUVE) spacecraft has achieved a resolution of $\lambda / \delta \lambda \approx$ 200 in the range $400-750 \AA$ in addition to providing 2-D images of the IPT in each line (Hall et al. 1994). While the fine structure of the EUV emissions remains blended, the higher resolution allows a comparison of the strengths of groups of lines within the multiplet. Table 1 shows the total emitted power from the IPT within several wavelength bands from a $610^{4} \mathrm{~s} E U V E$ exposure in March 1993 (Hall et al. 1994).

Hall et al. (1994) have generated a fit to the spectrum (integrated over the entire IPT) using a spectral fitting algorithm. By assuming constant electron temperature, they derived a composition. This paper reports the results of our initial attempts to model these data using a 3-D semi-empirical description of the IPT previously used to fit emissions at optical wavelengths (Thomas $1992 ; 1993 ; 1995)$. We concentrate, in particular, on four aspects of the observations; the possible influence of self-absorption, the observed east-west asymmetry, the spatial distribution of the emission, and the total emitted power. The results must be considered preliminary at this stage because further checking of the model (in particular the EUV rate coefficients) is required. We begin by describing briefly the model.

\section{The Model}

The semi-empirical model of the IPT, which has previously been described by Thomas (1992), solves the diffusive equilibrium equation for a multispecies plasma following the method of Bagenal (1985), giving the ion distribution along each field line based on the 
TABLE 1. Observed and modelled photon fluxes for several torus species

$\begin{array}{cccccc}\begin{array}{c}\text { Wavelength } \\ (\AA)\end{array} & \begin{array}{c}\text { EUV Emitted Power } \\ \text { Observed } \\ (\mathrm{W})\end{array} & \begin{array}{c}\text { Modelled } \\ (\mathrm{W})\end{array} & \begin{array}{c}\text { Difference } \\ (\%) \\ (\mathrm{W})\end{array} & \begin{array}{c}\text { Maximum } \\ \text { Modelled } \\ \text { Optical Depth }\end{array} & \begin{array}{c}\text { East-West } \\ \text { Asymmetry }\end{array} \\ \text { OII 426-434 } & 2.110^{9} & 3.110^{9} & 48 & 0.43 & \\ \text { OIII 505-512 } & 2.210^{9} & 5.110^{9} & 132 & 0.02 & 2.7 \\ \text { OII 536-543 } & 0.9810^{10} & 1.110^{10} & 12 & 0.26 & 2.5 \\ \text { SII 639-643 } & 4.010^{9} & 2.410^{10} & 500 & 0.95 & 2.4 \\ \text { SIV 654-665 } & 2.710^{10} & 4.310^{10} & 59 & 0.36 & 2.3 \\ \text { SIII 671-685 } & 1.010^{11} & 2.310^{11} & 130 & 1.32 & 2.3 \\ \text { SIII 697-705 } & 7.610^{10} & 6.910^{10} & -9 & 0.30 & 2.3 \\ \text { SIII 724-731 } & 8.710^{10} & 8.610^{10} & -1 & 0.27 & 2.3\end{array}$

plasma sciences (PLS) in situ measurements from Voyager I (Bagenal 1994). An offset tilted dipole (OTD) approximation of Jupiter's magnetic field was assumed. The ion temperatures were also obtained from PLS data $\left(T_{\|}=T_{\perp}\right.$ was assumed) while the electron temperature $\left(T_{e}\right)$ was taken from Sittler \& Strobel (1987). Suprathermal electrons have not been included. Taylor et al. (1995) have shown they contribute only a minor fraction to EUV emissions. For forbidden optical transitions, the emission from each volume element along the line of sight was computed by using a classical five-level equilibrium calculation following Osterbrock (1989). The integrated brightness was then computed by numerical integration assuming azimuthal symmetry about the OTD.

The model has been extended to include EUV emissions by incorporating higher levels in the model ions. Each model ion contains data for transitions between 15 to 35 levels. Rather than listing all levels and rate data, Table 2 simply gives the number of levels included for each ion and references to the transition probability and collision strength data for the observed EUV lines. In cases where data are only available for rates between LS levels, the fine structure rates for electric-dipole allowed transitions have been obtained by scaling the total rate by the relative line strength according to the rules of Russell-Saunders coupling (Condon \& Shortly 1970) and the fine-structure rates for forbidden transitions have been obtained by scaling by the state degeneracies.

\section{Optical Depth Effects in the EUV}

Strobel \& Davis (1980) noted that several of the transitions making up the EUV spectrum of the IPT are short-lived and that self-absorption needs to be considered. They suggested that optical depths as high as 3 could occur in the IPT. Shemansky \& Smith (1981) pointed out that whilst the $\mathrm{O}^{+} / \mathrm{O}^{2+}$ transitions at $833 \AA$ should not be influenced by self-absorption, it was possible that the $S^{2+}$ transitions near $680 \AA$ might be affected. However, they suggested that the absence of measurable changes in the $680 / 833 \AA$ line ratio scanning across the IPT (Shemansky \& Sandel 1981) provided an upper limit for both self-absorption $(<10 \%)$ and the column density of $\mathrm{S}^{2+}\left(5.510^{12}\right.$ $\mathrm{cm}^{-2}$ ). The limit on the column density was made assuming an ion temperature of 26 $\mathrm{eV}$. The question of optical depth effects has received almost no attention since these early papers.

The model can be used to determine the column density of $\mathrm{S}^{2+}$ in the IPT. The value of this quantity at the western ansa when the torus is seen edge-on is $1.9710^{13} \mathrm{~cm}^{-2}$ exceeding the limit set by Shemansky \& Smith (1981) by 4 . 
TABLE 2. Atomic data

\begin{tabular}{lccll} 
Ion & No. of levels & $\lambda$ & A-value & \multicolumn{1}{c}{$\Omega$} \\
OII & 19 & $426-434$ & WSG & LM \\
& & $536-543$ & HH1 & I \\
OIII & 25 & $505-512$ & NS & I \\
SII & 31 & $639-643$ & BD & M \\
SIII & 27 & $671-685$ & S & HH2 \\
& & $697-705$ & HH2 & HH2 \\
& & $724-731$ & HH2 & HH2
\end{tabular}

REFERENCES- WSG = Wiese et al. (1966); LM = Landini \& Monsignori Fossi (1990); HH1 $=$ Ho \& Henry (1984a); I = Itikawa et al. (1983); NS = Nussbaumer \& Storey (1981); BD = Bates \& Damgaard (1949); $M=$ Mewe (1972); RRC = Ryan et al. (1989); HH2 = Ho \& Henry (1984b); S = Sultana (1993).

In order to assess the influence of self-absorption, we have included this effect in the model. Even if transitions are optically thick in the plane of the torus, the optical depth out of the plane is always small, so that resonance line trapping will not affect level populations. The derived populations are then used to obtain the emissivity, $\eta$, and the opacity, $\chi$. The final intensities are obtained by integrating the radiation transfer equation along the line of sight. Between any two depths, $\tau_{1}$ and $\tau_{2}$, the intensity, $I$, at frequency, $\nu$, is related by

$$
I\left(\tau_{1}, \nu\right)=I\left(\tau_{2}, \nu\right) e^{\tau_{2}(\nu)-\tau_{1}(\nu)}+\int_{\tau_{1}(\nu)}^{\tau_{2}(\nu)} S(\nu, t) e^{t-\tau_{1}(\nu)} d t
$$

where $S(\nu)$ is the source function, $\eta(\nu) / \chi(\nu)$.

The $\mathrm{S}^{2+} 678 \AA$ transition between the $3 \mathrm{p} 3 \mathrm{~d}^{3} \mathrm{D}_{1}^{o}$ and the ground state, $3 \mathrm{p}^{2} 2{ }^{3} \mathrm{P}_{0}^{e}$ has the largest collision strength and transition probability $\left(8.910^{9} \mathrm{~s}^{-1}\right)$ in the EUV wavelength range. The optical depth at line centre is

$$
\tau_{0}=2.6\left(\frac{N_{i}}{10^{13}[\mathrm{~cm}]}\right)\left(\frac{10^{5}[K]}{T_{i}}\right)^{1 / 2}
$$

where $N_{i}$ and $T_{i}$ are the $\mathrm{S}^{2+}$ column density and ion temperature, respectively.

Figure 1 shows the optical depth of all $S^{2+}$ lines between $670 \AA$ and $710 \AA$ at the point of maximum brightness on the western ansa of the torus. The observer sees the torus edge-on. An optical depth of 1.3 in the $\mathrm{S}^{2+} 678 \AA$ transition is evident. Several other transitions are also affected. The total flux from the $680 \AA$ multiplet is reduced by $15 \%$ at this point in the torus compared to the optically thin case. From Figure 1 that the $680 / 700 \AA$ line ratio must also be reduced because of the lower optical depth of the 700 A multiplet.

To illustrate this further, simulated images of the torus have been produced at several wavelengths. Five examples are shown in Figure 2. The two lower panels ( $d$ and $e$ ) show the appearance of the torus at $680 \AA$ including and ignoring the effect of optical depth (all lines in this multiplet have been summed). Visually, the general appearance of the torus remains unaltered but quantitatively there are some changes. Over a projected area of $3.2510^{11} \mathrm{~km}^{2}$ centred on Jupiter, the observed intensity in the $675-686 \AA$ band 


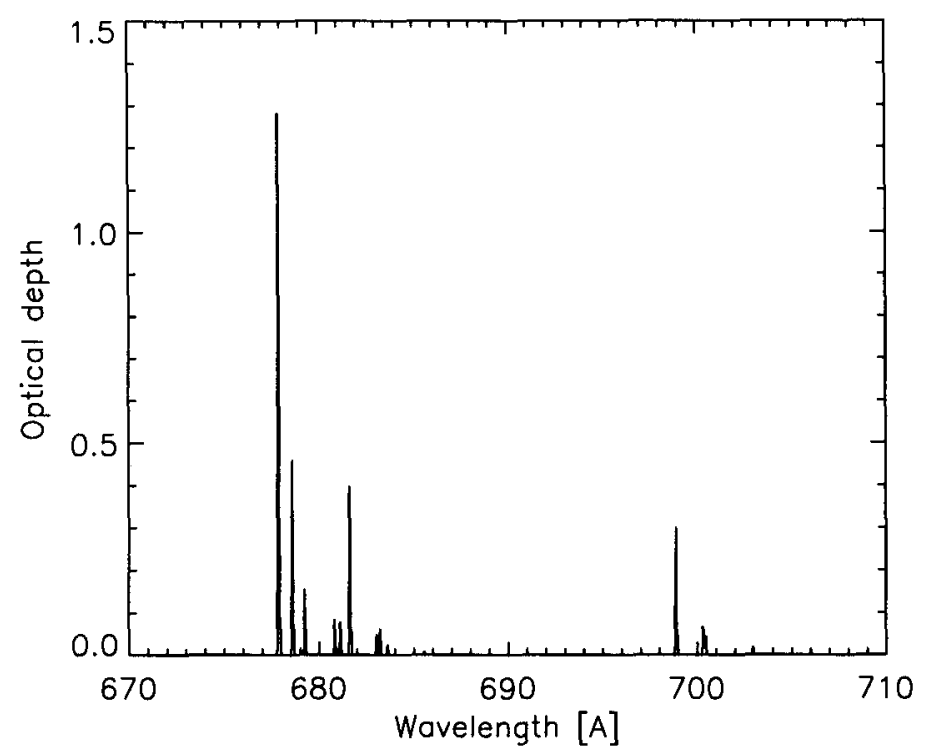

FIGURE 1. Optical depth of SIII lines near $700 \AA$

is reduced by $7 \%$ because of optical depth effects. The effects are most noticeable at the ansae. The $697-705 \AA$ band is reduced by $0.5 \%$ for the same observing geometry. It may be interesting to look for spatial and temporal variations in the $680 / 700 \AA$ ratio and whether they correlate with total SIII intensity.

Although these calculations indicate that self-absorption will be most significant for the $\mathrm{S}^{2+} 680 \AA$ line, Table 1 shows that lines within other multiplets can also reach high values. Caution should be exercised when treating the SII $641 \AA$ and the OII $430 \AA$ multiplets.

\section{The East-West Asymmetry}

Sandel \& Broadfoot (1982) noted that UVS data showed IPT EUV emissions to be asymmetric about Jupiter, being brighter at the western ansa than at the eastern. Barbosa \& Kivelson (1983) and Ip \& Goertz (1983) suggested that this was the result of a dawn-dusk electric field. The field shifts the centre of the IPT towards the east as seen from the Earth leading to compression of the plasma at the dusk ansa and expansion at the dawn ansa. The expansion cools the plasma at the dawn ansa (relative to Voyager 1 in situ measurements at the dusk ansa) giving lower volume emission rates.

The model includes the effect of the dawn-dusk electric field following the method described in Smyth \& Combi (1988). Schneider \& Trauger (1995) have presented evidence that this description is not adequate to explain the short timescale oscillation of the western ansa. However, the temporal resolution of $E U V E$ is insufficient to resolve this motion and therefore the formulation of Smyth \& Combi is adopted as a first approach.

The method allows the determination of first order corrections to the ion and electron 
densities and temperatures using a single independent variable $\epsilon$, the so-called "shift parameter," which describes the ratio of the dawn-dusk electric field strength to that of the corotation field. Smyth \& Combi (1988) relate the densities and temperatures to the western (dusk) ansa which was investigated by the Voyager 1 in situ measurements.

The shift of the IPT with respect to Jupiter and the difference in brightness between the two ansae as seen from the Earth, have been measured on several occasions at optical wavelengths (e.g. Morgan, 1985). Values in the range $\epsilon=0.03 \pm 0.01$ have been found.

The influence of the dawn-dusk electric field is clearly evident in EUVE images. Hall et al. (1994) found observed brightness ratios of $2.3 \pm 0.3$ and $2.7 \pm 0.5$ for SIII $680 \AA$ and OII $539 \AA$, respectively, for the regions between 5 and $6 R_{J}$ on either side of the planet. Using $\epsilon=0.03$, the model predicts brightness ratios of 2.4 (SIII $680 \AA$ ) and 2.5 (OII 539 $\AA)$, in excellent agreement with observation.

The slight difference in the magnitude of the asymmetry for the two lines is the result of the slightly higher excitation energy of OII. Optical depth effects are not major contributors to the observed difference in the ratio between the two species. This is, in part, because the reduction in the ion density at the eastern ansa is partially compensated for by the decrease in ion temperature (see Eq. (2)).

Figure 2 shows the appearance of the IPT in both OII $539 \AA$ and SIII $680 \AA$. In all cases, the IPT is seen edge-on. Table 1 gives the predicted east-west asymmetry for 8 EUV lines based on $\epsilon=0.03$. It should be noted that the modelled brightness ratio in the EUV is highly sensitive to $\epsilon$ and that values outside the range $0.025<\epsilon<0.035$ are clearly incompatible with the east-west ratios reported by Hall et al. (1994).

\section{The Spatial Distribution of Emission}

The steep rise in $T_{e}$ with radial distance in the IPT (Sittler \& Strobel 1987) has led to a misconception that the maximum emission at EUV wavelengths must come from regions well outside Io's orbit and up to $7 \mathbf{R}_{J}$ from Jupiter (e.g. McGrath et al., 1995). Figure 2 shows that the maximum emission from the IPT actually occurs close to $6 \mathbf{R}_{J}$ for the major species. This is simply because the effect of the rise in $T_{e}$ with radial distance is more than compensated for by the sharp decrease in density. Thus, despite the fact that only the tail of the electron distribution excites EUV emissions, density effects dominate. The rise in $T_{e}$ does push the maximum emission out slightly beyond the position of the maximum at visible wavelengths (the "ribbon"). However, EUVE, which has a spatial resolution of about $1 \mathrm{R}_{J}$, should not be able to resolve the difference.

An exception to this behaviour is the OIII $509 \AA$ emission which peaks at $7.2 R_{J}$ in the current model. The extended width of the OIII emission should, in principle, be detectable by $E U V E$ and will be the subject of further investigations. The appearance is caused by the increasing mixing ratio of OIII with jovicentric distance (see Bagenal et al., 1992). This remarkable extension of the emission also leads to an unusual modelled east-west asymmetry ratio at this wavelength because of the restriction of the calculation to the region between 5 and $6 \mathbf{R}_{\boldsymbol{J}}$ (see Table 1).

\section{The Total Emitted Power}

The model has been used to compute the total emitted power from the IPT within 8 wavelength bands which include major emission features. The results are compared to the observed values in Table 1 and the difference between the predicted and observed intensities is given as a percentage of the observed. The results show significant similarities and discrepancies. It should be noted at this point that while errors in the observed fluxes are, in most cases, less than $10 \%$, the errors in the emission rate coefficients for EUV 


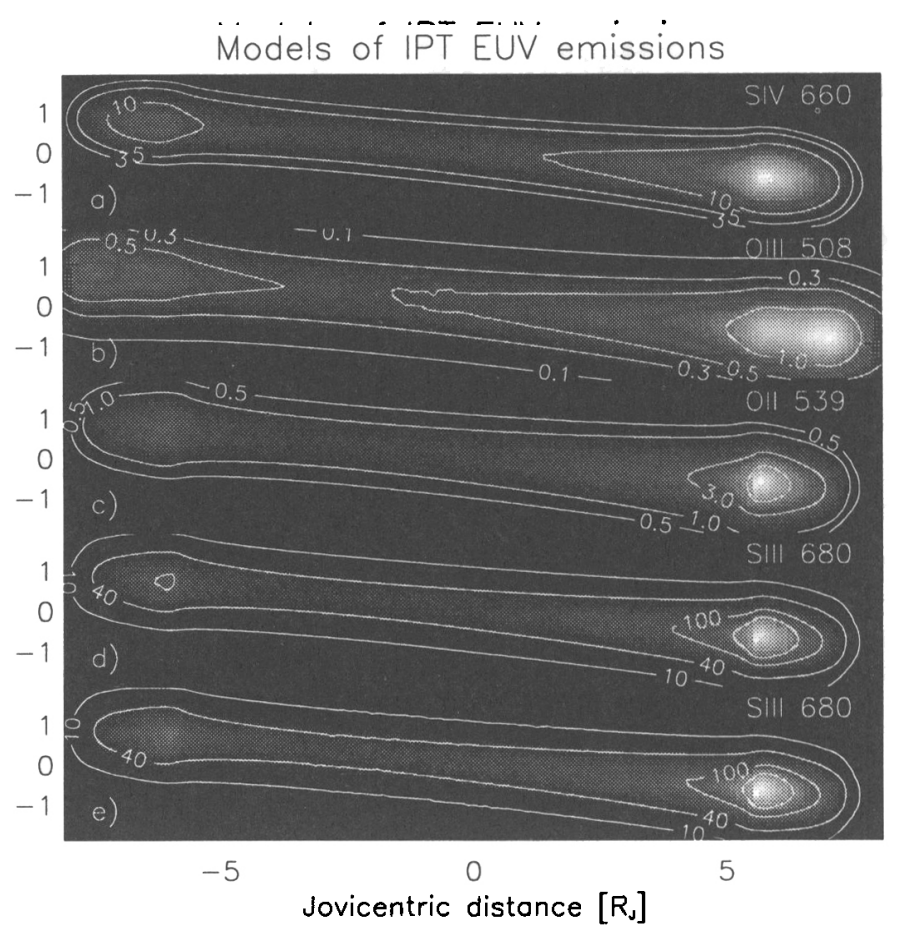

FIGURE 2. Models of the spatial distribution of IPT EUV emissions: (a) SIV $660 \AA$, (b) OIII $508 \AA$, (c) OII $539 \AA$, (d) SIII $680 \AA$ excl. optical depth effects, (e) SIII $680 \AA$ incl. optical depth effects.

transitions often far exceed this. Hence, it is not straightforward to determine whether the IPT was either (a) strictly "Voyager-like" at the time of the EUVE observations, (b) significantly different from Voyager times or (c) diagnosed incorrectly from Voyager measurements.

Bearing this point in mind, the most satisfactory result is for OII $539 \AA$ for which observed matches predicted at the $10 \%$ level. OII is now thought to be the most abundant species in the IPT. The Voyager 1 PLS experiment was unable to differentiate between OII and SIII because of their identical mass per charge ratios while the UVS experiment could not separate OII from OIII because of the almost identical wavelengths of their emissions at $833 \AA$. The excellent agreement between the current Voyager model and the $E U V E$ data suggests that this difficulty has been correctly resolved. There remains, however, the OII $430 \AA$ multiplet which is significantly weaker than predicted although the collision strengths for this line are poorly known.

Two of the three SIII multiplets are well modelled. However, the model of the $680 \AA$ emission is more than a factor of two too high. The similarity in the excitation energies of these three multiplets indicates that these lines should be in a simple ratio determined by their thermally averaged collision strengths. This, in turn, implies that if the observed ratios for the three SIII multiplets are correct, the atomic data source for the model must 
be wrong. It should be noted that we have used theoretical calculations for these lines while the COREQ program used to analyse Voyager UVS data (e.g. Shemansky, 1988) scales theoretical values to match experimental data. The resolution of this problem remains the subject of further work.

The $60 \%$ difference between model and observation for the SIV emission at $660 \AA$ is considered to be a good result given the uncertainty in the mixing ratio of this minor species. This is also true to a lesser extent of the OIII emission at $509 \AA$. The model suggests that emission at high jovicentric distance dominates at this wavelength. This clearly needs to be investigated but it appears that a reduced mixing ratio near $7 R_{J}$ would bring the model into closer agreement with observation.

The SII $641 \AA$ emission shows by far the largest disagreement probably because of the assumed emission rate coefficients which have only been crudely estimated so far.

\section{Conclusion}

The good agreement in several areas between observations and the model shows this to be a good starting point for the detailed modelling of $E U V E$ observations of the IPT. There remain points where further work needs to be done. In particular, verification and checking of the emission rate coefficients is urgently required, especially for SII. However, the successful description of the east-west asymmetry shows that this approach will be very useful in deriving the properties of the Io plasma torus.

N. Thomas would like acknowledge useful discussions with D.T. Hall, M.A. McGrath, and M. Taylor during the International Jupiter Watch Torus Discipline workshop in Las Cruces, New Mexico, April 26-27, 1995. R. Lieu acknowledges the support of NASA contract NAS5-30180.

\section{REFERENCES}

Bagenal, F. 1985, Plasma conditions inside Io's orbit: Voyager measurements, J. Geophys. Res., 90, 311

BAgenal, F. 1994, Empirical model of the Io plasma torus: Voyager measurements, J. Geophys. Res., $99,11,043$

Bagenal, F., ET AL. 1992, The Abundance of $\mathrm{O}^{++}$in the Jovian Magnetosphere, Geophys. Res. L., 19, 79

Barbosa, D. D. \& M. G. Kivelson 1983, Dawn-dusk electric field asymmetry of the Io plasma torus, Geophy. Res. L., 10, 210

BAtes, D. R. \& A. DAmgaARD 1949, The calculation of the absolute strengths of spectral lines, Phil. Trans. Roy. Soc. London, 242, 101

BRoADFOOT, A. L., ET AL. 1979, Extreme ultraviolet observations from Voyager I encounter with Jupiter, Science, 204, 979

Condon, E. U. \& G. H. Shortly 1970, The theory of atomic spectra, Cambridge: Cambridge University Press

HALL, D. T., Er AL. 1994, Extreme Ultraviolet Explorer satellite observation of Jupiter's Io plasma torus, ApJL, 426, L51

Ho, Y. K. \& R. J. W. HenRY 1984a, Oscillator strengths for OII ions, J. Quart. Spectrosc. Radiat. Transfer, 31,57

Ho, Y. K. \& R. J. W. HENRY 1984b, Oscillator strengths and collision strengths for S III, ApJ, 282, 816

IP, W. -H. \& C. K. GoERTz 1983, An interpretation of the dawn-dusk asymmetry of UV emission from the Io plasma torus, Nature, 302, 232 
Itikawa, Y., S. Hara, T. Kato, S. Nakazaki, M. S. Pindzola, \& D. H. Crandall 1983, Recommended data on excitation of carbon and oxygen ions by electron collisions, Institute of Plasma Physics, Nagoya University

LANDini, M. \& B. C. Monsignori, Fossi 1990, The X-UV spectrum of thin plasmas, A\&AS, Ser., 82, 229

McGrath, M. Ex AL. 1995, Response of the Io Plasma Torus to Comet Shoemaker-Levy 9, Science, 267,1313

MEwE, R. 1972, Interpolation Formulae for the Electron Impact Excitation of Ions in the H-, $\mathrm{He}-, \mathrm{Li}-$, and Ne-Sequences, A\&A, 20, 215

MoRgaN, J. S. 1985, Temporal and spatial variations in the Io torus, Icarus, 62, 389

Nussbauen, H. \& P. J. STOREY 1981, O III: Intercombination and Forbidden Lines., A\&A, 99,177

OSTERBRock, D. E. 1989, Astrophysics of Gaseous Nebulae and Active Galactic Nuclei, Mill Valley, Calif.: Science Books

Ryan, L. J., L. A. RAYBURN, \& A. J. CunNINGHAM 1989, Measurements of oscillator strengths for EUV emissions of ionized oxygen, nitrogen and sulfur, J. Quant. Spectros. Radiat. Transfer, 42, 295

SANDEL, B. R. \& A. L. BROADFOOT 1982, Io's hot plasma torus-A synoptic view from Voyager, J. Geophys. Res., 87, 212

Schnemer, N. M. \& J. T. Trauger 1995, The Structure of the Io Torus, ApJ, in press

SHEMANSKY, D. E. 1988, Energy branching in the Io plasma torus: The failure of neutral cloud theory, J. Geophys. Res., 93, 1773

Shemansky, D. E. \& B. R. SANDEL 1981, The injection of energy into the Io plasma torus, J. Geophys. Res., 87, 219

SHEMANSKY, D. E. \& G. R. SMITH 1981, The Voyager 1 EUV spectrum of the lo plasma torus, J. Geophys. Res., 86, 9179

SitTLER, E. C. \& D. F. STroBel 1987, Io plasma torus electrons: Voyager I., J. Geophys. Res., 92,5741

SMYTH, W. H. \& M. R. CoMBI 1988, A general model for Io's neutral gas clouds II: Application to the sodium cloud, ApJ, 328, 888

STrobel, D. F. \& J. Davis 1980, Properties of the Io plasma torus inferred from Voyager EUV data., ApJL, 238, L49

Sultana, N. NaHAR 1993, Transition Proabilities for Dipole Allowed Fine Structure Transitions in Si-like Ions: Si I, S III, Ar V and Ca VII, Physica Scripta, 48, 297

TAYLOR, M. H. ET AL. 1995, A comparison of the Voyager 1 UVS and PLS measurements of the Io Plasma. Torus, J. Geophys. Res., submitted

Tномаs, N. 1992, Optical observations of Io's neutral clouds and plasma torus, Surv. Geophys., 13,91

Thomas, N. 1993, Detection of [OIII] 55007 emission from the Io plasma torus, ApJL, 414, L41

Thomas, N. 1995, Ion temperatures in the Io plasma torus, J. Geophys. Res., 100, 7925

Wiese, W. L., M. W. Smith, \& B. M. Glennon 1966, Atomic Transition Probabilities, 1, NSRDS-NRS 22 